\title{
Author Correction: Characterization of a filovirus (Měnglà virus) from Rousettus bats in China
}

Xing-Lou Yang, Chee Wah Tan, Danielle E. Anderson (D), Ren-Di Jiang, Bei Li, Wei Zhang, Yan Zhu, Xiao Fang Lim, Peng Zhou, Xiang-Ling Liu, Wuxiang Guan, Libiao Zhang, Shi-Yue Li, Yun-Zhi Zhang, Lin-Fa Wang (D) and Zheng-Li Shi

Correction to: Nature Microbiology https://doi.org/10.1038/s41564-018-0328-y, published online 7 January 2019.

In the version of this Letter originally published, in the 'Phylogenetic analysis' section of the Methods, the authors mistakenly stated that the GenBank accession number for the Ravn virus genome sequence was FJ750958. The correct accession number is DQ447649 for Ravn virus, Kenya, 1987. Accordingly, the label 'RAVN2007' in Fig. 1b should have been 'RAVV1987'. This mistake does not change any conclusions in this study. This statement and figure have now been amended in all versions of the Letter, and the Supplementary Information file has been updated accordingly. 